\title{
Helicobacter Pylori Eradication - A New Look at Old Problems
}

\author{
Morozov IA* \\ Chumakov Federal Scientific Center for research and development of immune-and-biological products of Russian Academy of Sciences, Russia
}

*Corresponding author: Morozov IA, Chumakov Federal Scientific Center for research and development of immune-and-biological products of Russian Academy of Sciences, Russia

Submission: 監 December, 2018; Published: 些February 14, 2019

\begin{abstract}
Summary
A detailed critical analysis of the "Treatment of H. pylori infection" from all Maastricht agreements is presented in the dynamics of their improvement since 1996. It is shown that no significant changes occurred in these recommendations in either drugs used for eradication therapy, either in regimens treatment or in its results during the 20-year period. It is offered to refuse completely to use antibiotics that have been discredited (clarithromycin, metronidazole etc.) in favor of using bactericidal preparations. It is necessary to radically review the procedure of treatment and switch from tablets to "liquid" technology, which allows acting directly on the infection throughout the surface of the gastric mucosa.
\end{abstract}

Keywords: Helicobacter pylori; Eradication; Chronic gastritis; Peptic ulcer disease

\section{Introduction}

The history of recommendations for the diagnosis and treatment of diseases associated with Helicobacter pylori (Hp) has been around for more than 20 years, beginning with the appearance in 1994 of the agreed recommendations of the US National Institutes of Health, which recognized the need for anti-Helicobacter therapy in patients with peptic ulcer [1] . The first European guidelines were adopted in the small Dutch town of Maastricht in 1996 and became the first standardized and consistent guidelines for the diagnosis and treatment of patients in the European Region who have Hp infection in the stomach. "Maastricht Consensus" [2] was prepared by a group of experts led by Malfertheiner P, Megraud F, 0 Morain C, Graham D. Who formed, led, and still lead the European Helicobacter Study Group (EHSG). These recommendations were developed on the basis of literary data based on scientific evidence, expert opinions and medical practitioners. The recommendations presented all aspects of the problem of Helicobacter pylori (Hp) infection: methods for diagnosing Hp infection, strict and recommended indications for eradication, even taking into account the wishes of the patient, as well as various options for eradication therapy. It is the problem of eradication of $\mathrm{Hp}$ infection that has been and still is key in this issue.

In the period since 1983, that is, from the time BJ Marshall and $\mathrm{R}$ Warren received objective evidence of all three "Koch postulates" for Hp infection, a large number of open clinical trials on eradication of the infective were published. The analysis of the futility of $\mathrm{Hp}$ eradication using monotherapy with bismuth, amoxicillin, clarithromycin, metronidazole, even with long-term (28 days) therapy, as well as the absence of any reaction from $\mathrm{Hp}$ infection to the equally long-term use of $\mathrm{HCl}$ secretion inhibitors (famotidine and omeprazole ). All these data are analyzed in detail in the monograph "Helicobacter pylori infection. Aspects of pathogenesis and therapy"[3]. The Brocades Pharma Yamanouchi Group (Netherlands), published under the auspices of the pharmaceutical company, presents an analysis of the results of more than 100 papers showing the futility of $\mathrm{Hp}$ eradication when using combinations of bismuth preparations (28 days) with metronidazole, amoxicillin, tetracycline and clarithromycin (10-14 days). Only the combination of bismuth with two antibiotics made it possible to achieve acceptable levels of eradication above $80 \%$. But due to the cautious attitude of the medical community to bismuth preparations in the Maastricht consensus, an effective course of anti-Helicobacter therapy was recommended, which included the use of a proton pump inhibitor (PPI) and two antibiotics, taking into account the probable resistance of microorganisms to them. A seven-day course of treatment was considered adequate, and in case of failure of the first-line therapy course, before prescribing secondline drugs, endoscopy, isolation of a pure culture and determination of its sensitivity to antibiotics were recommended. The Maastricht Consensus of 1996 had a great impact on the treatment of Hpinfected patients and accelerated the implementation of eradication therapy.

However, primary care physicians, for whom, in fact, recommendations were created, remained unclear questions regarding treatment approaches, indications for $H$. pylori eradication, and therapeutic regimens. Therefore, the first original recommendations, in order to create a more comprehensive 
practical guide to modern treatment of patients with Hp infection for use in clinical practice, were revised and supplemented in 2000 in the Maastricht II consensus report [4]. The treatment regimen recommended by Maastricht II was called the "triple therapy". It is based on the use of two antibiotics (mainly clarithromycin and amoxicillin) and IPP in the standard dose twice a day in a short seven-day course. However, no antimicrobial regimen in controlled studies provided a $100 \%$ level of eradication. At the time of Maastricht II, the average European level of $\mathrm{H}$. pylori eradication with the use of triple therapy was $80-85 \%$. Therefore, in cases of unsuccessful first-line treatment, clinicians were immediately asked to plan a second line, the most optimal of which is "quadrotherapy" (PPI, bismuth, tetracycline, and metronidazole). That is, the main principle of treatment established in Maastricht II is that the first and second line eradication schemes were considered together.

The recommended second-line regimen is usually successful in case of primary inefficiency of clarithromycin-containing drugs, but does not always help in case of primary inefficiency of metronidazole-containing agents. Therefore, the choice of the second line of treatment has always depended on the initial treatment. If clarithromycin-containing substances were used first, then metronidazole-containing preparations were used as a second line of treatment as a method of choice. An integral consequence of the therapeutic strategy was that during initial treatment it was undesirable to combine clarithromycin and metronidazole. Maastricht II highly recommended a combination of PPI, clarithromycin and amoxicillin for first-line therapy. This combination is preferable to the combination of PPI, clarithromycin and metronidazole.

In 2005, new recommendations were adopted in Florence - Maastricht III [5]. By the time this consensus was adopted, the indicators of $\mathrm{Hp}$ resistance to a number of drugs had significantly increased in the world. Thus, indicators of resistance of microorganism strains to metronidazole were within $19-42 \%$ in European countries and $33-37 \%$ in the USA, and more than $50 \%$ in developing countries (including Ukraine). For clarithromycin, these figures were about $13 \%$ in the United States and Japan. In European countries, they varied widely: relatively low in the countries of Northern $(4.4 \%)$ and Central $(8.7 \%)$ of Europe, and, on the contrary, high (24\%) in the countries of Southern Europe. Indicators of resistance to amoxicillin and tetracycline were very low and had no clinical significance.

The first-line eradication scheme, as before, remained a triple scheme. It was again emphasized that in this scheme metronidazole is used instead of amoxicillin, if the prevalence in the population of Hp-resistant strains in metronidazole is less than $40 \%$. In Ukraine, metronidazole in the ternary scheme was not recommended for use at all. In addition, the Maastricht-III consensus introduced an amendment stating that the ternary scheme should not be prescribed if clarithromycin's resistance to Helicobacter pylori in a specific region exceeds $20 \%$. At the same time, the experts listened to the opinion of a number of their colleagues that the first-line therapy scheme should provide the highest efficacy of treatment.
In the recommendations it was written that an alternative to triple regimens may be "second-line" therapy - quadrotherapy, but with an extremely unsuccessful regimen of medication: PPI in a standard dose 2 times/day, bismuth subsalicylate/subcitrate 120mg 4 times/day, metronidazole 500mg 3 times/day, tetracycline $500 \mathrm{mg}$ 4 times/day. The course of treatment is 7-14 days. It was stated that the 14-day schemes are not much, but still more effective $(12 \%$ difference) compared to the 7-day schemes. However, despite the recommendations, in most countries, 14-day therapy remained economically unprofitable.

In November 2010, an meeting of an international group of experts was held again in Florence (Italy), which reviewed the next changes in the Recommendations on the diagnosis and treatment of diseases associated with Hp for the European Union countries, in which significant attention was paid to optimizing antihelicobacter therapy regimens [6]. The Maastricht-IV consensus recommendation supplemented the proposal to conduct antihelicobacter therapy in patients with idiopathic thrombocytopenic purpura, iron deficiency anemia of unknown etiology and B12deficiency anemia.

For the eradication of $\mathrm{Hp}$, a number of first and second line schemes have been proposed. According to international recommendations, the effectiveness of eradication schemes should be at least $80 \%$. However, this level of efficiency of eliminating infection has been criticized by specialists who believed that, like other infections, this indicator should be no less than $90 \%$. Be that as it may, the effectiveness of the majority of traditionally used eradication therapy regimens significantly decreased and did not reach $80 \%$. As a first-line treatment regimen, as in the previous consensus, a triple was most often used, consisting of a combination of a proton pump inhibitor (PPI) and two antibiotics, clarithromycin, metronidazole or amoxicillin. Meta-analyzes and systematic reviews showed that in the overwhelming majority of cases, the triple therapy efficacy rate is unacceptably low - only in $18 \%$ of cases it exceeded $85 \%$ and in about $60 \%$ of studies did not reach $80 \%$ [7]. It was assumed that the reasons for the decrease in the effectiveness of treatment are the formation of antibiotic resistance to HP (acquired antibiotic resistance) and insufficient patient compliance - non-compliance with the dosages, regimen and duration of medication. Given all the above, the MaastrichtIV consensus retained the Maastricht-III recommendation on the possibility of using quadrate therapy (PPI, tetracycline, metronidazole and bismuth), previously a second-line therapy, as the main treatment regimen.

Attempts to introduce a consistent 10-day and combined sequential-simultaneous therapy regimens did not succeed, since their effectiveness was highly questionable. In addition, to increase the effectiveness of eradication therapy, it was suggested: a further increase in the duration of treatment up to 10-14 days, the inclusion of reserve antibiotics in the treatment regimens, the use of three antibiotics, increased suppression of acid production, and the addition of antibiotics and probiotics to the treatment regimens. 
In this state, the Maastricht veterans approached the need for a regular meeting in Florence (Italy) to review the recommendations for combating Hp infection for doctors of the European Union. The Maastricht-V consensus was held in 2015. The main provisions of the consensus were reported at the $19^{\text {th }}$ International Conference on Helicobacter pylori infection and microbiota in September 2016 in Magdeburg (Germany). Finally, in January 2017, as usual with a two-year delay, they were published in the journal Gut [8]. Traditionally, the main purpose of this conciliation meeting was to systematize and present, from the standpoint of evidence-based medicine, data on Hp-associated diseases accumulated over the past few years, including questions of its diagnosis and treatment. In this article, we present the main provisions of the Maastricht- $V$ consensus on only the eradication therapy for Hp infections.

The consensus section on eradication therapy reiterates the position of Maastricht IV that Hp resistance to antibacterial drugs tends to increase in all regions of the world. However, the cited data practically does not differ much from the previous recommendations, except for amazing figures for the African continent, in which the resistance to amoxicillin for 2015 was $17.8 \%$. It has long been known that Hp resistance in this drug is not produced, and the naturally occurring Amoxicillin-resistant $\mathrm{Hp}$ strains are extremely rare, as confirmed by indicators on all other continents of the globe. If the determination of culture and standard sensitivity to antimicrobial drugs in vitro is carried out, then this absolutely does not reflect the situation in vivo, when resistance is judged by the result of eradication therapy obtained in the group treated according to a specific scheme.

Experts of the EU countries, apparently, together with pharmaceutical companies continue to recommend all the same antibiotics (clarithromycin, metronidazole, tetracycline), which began to be used at the beginning of the «Helicobacter era» from the mid 80s of the last century. During this time, not a single specific means for Hp eradication has been created, and only amoxicillin of the drugs used deserves attention.

At its core, the Maastricht-V recommendations are practically the same as the Maastricht-IV consensus. With regard to the duration of first-line eradication therapy, instead of the possible alternativeness of quadropathy with or without bismuth preparations, the term "should" is used for 14 days, unless local effectiveness has been proven for 10 days. The possibility of using the second and even third-line therapy, the use of higher doses of PPI, the simultaneous, sequential or combined simultaneoussequential regimens are also approved.

Above, we have already mentioned that the meta-analyzes carried out for the entire period after the development of the Maastricht agreements show a rather low level of efficiency in the eradication of $\mathrm{Hp}$ infection. In more than half of the work, it did not reach $80 \%$, and this is despite the fact that the main reasons are considered to be increased resistance of the infective to the antibiotics used, a violation by patients of the regimen and duration of the drugs, as well as non-compliance with their dosages. The exclusion of such cases of "per protocol" artificially increased the effectiveness of eradication therapy. It was repeatedly mentioned that in urgent therapy, the effectiveness of eradication is significantly lower than indicated in meta-analyzes and, in most cases, does not exceed $50-60 \%$ in any treatment regimens.

As an example, a unique study of Japanese gastroenterologists, published in the Journal of Gastroenterology in 2000 [9], can be cited. This study was attended by 138 specialized departments and clinics of 15 leading universities in Japan. In a randomized doubleblind study of several hundreds of patients with gastric ulcer and 12 duodenal ulcer, they studied the effect of supportive therapy with histamine $\mathrm{H} 2$ receptor antagonists on the occurrence of exacerbations of the disease within 12 months after triple first-line therapy with lansoprazole (6-8 weeks) and clarithromycin with amoxicillin (7 days).

Before treatment, the presence of Hp infection was determined by bacteriological, urease, and histological tests. Hp infection with gastric ulcer was $87 \%$, and with duodenal ulcer $91 \%$. After therapy, the determination of the presence of $\mathrm{Hp}$ infections was not performed, considering that the effectiveness of eradication is 98\% with reference to German researchers [10] who used a similar triple therapy. As a result of one-year follow-up, it was found that maintenance therapy has virtually no effect on the number of exacerbations: with gastric ulcer with supportive therapy, the number of exacerbations was $25 \%$, without it - $27 \%$, while with duodenal ulcer $39 \%$ and $43 \%$, respectively. Indirectly, these figures indicate a very low efficacy of eradication therapy and could in no way be a consequence of reinfection.

There are objective reasons that reduce the effectiveness of modern schemes for the treatment of acid-related diseases during Hp eradication in the stomach using tablet preparations on an empty stomach. First, over the years, the same antibiotics used to treat pneumonia, urogenital infections, but not specific for $\mathrm{Hp}$ infections in the stomach, have been used. Secondly, the advantages of proton pump inhibitors (PPIs) are unjustifiably hypertrophied over histamine H2-receptor antagonists when used in treatment regimens as «basic preparations». They are even attributed the properties of synergy with clarithromycin during Hp eradication [11], although this is nothing but a manifestation of the protection of an antibiotic that is not resistant in an acidic environment against inactivation and decay [12].

PPIs in clinical and outpatient practice are used almost without preliminary testing of the sensitivity of patients to this class of drugs, despite the fact that about $20 \%$ of the population of people are insensitive to them, and $70 \%$ of them have "nightly acid breakthroughs" [13]. Such a situation may deliberately reduce the upper limit of eradication efficiency below $80 \%$ when used in the schemes of clarithromycin, which is quickly inactivated in an acidic environment. By virtue of this, the use of clarithromycin in ternary schemes with the use of De-Nol as a base preparation instead of hydrochloric acid secretion inhibitors, as some researchers suggest, becomes meaningless [14]. Checking the efficacy of similar schemes shows an extremely low result of eradication. 
It has repeatedly been shown that four-part regimens are more effective in the eradication of $\mathrm{Hp}$ infection. At the turn of the century, proposals were made more than once to use such schemes as a "first line" therapy, even without any extension of its terms [1517]. Experts of the European Group for the Study of Helicobacter pylori, until recently, rejected such proposals. The only argument of opponents of such an approach was the statement that the application of four-component schemes is accompanied by a large number of undesirable side effects [11]. However, it is possible to form such four-part schemes, with the use of which the number of side effects of therapy will be minimal.

Third, the fact of persistence of the infecte in the depth of the mucus wall layer, the hydrophobic surface of which prevents drug penetration into this zone, is not taken into account $[18,19]$. The mucous layer is not an obstacle for $\mathrm{Hp}$, since it serves as a medium of obscuration. Even with adhesion to the surface of the apical membrane of the epithelium of the stomach, the remaining surface of the bacterium is immersed in the mucin gel of the mucous layer. In the depths of this layer, Hp selects zones with a $\mathrm{pH}$ of 6-7, at which it can actively reproduce. The Hp bacteria in the layer partly disappear together with the rejecting mucus and are later found in the feces of infected patients. The bacteria remaining in the mucus layer move to the optimum $\mathrm{pH}$ zone due to chemotaxia.

Fourthly, many doctors still believe that anti-helicobacter drugs act by absorbing them in the small intestine and creating high concentrations in the tissues of the stomach or after eckretsii in his lumen. However, a drug such as clarithromycin, originally developed for the treatment of pneumonia, has the highest concentrations in the tissues of the lungs, while in the tissues of the stomach their concentration is four times lower [20], and the excretion in the gland does not exceed $1 \%$ from the administered dose. It follows from this that the destruction of the infective can be achieved only by directly acting with bactericidal preparations inside the stomach over the entire surface of its mucosa. It is impossible to achieve such an effect through eradication with tablet forms of drugs, no matter how strong they are.

It should be particularly noted the opinion of pharmacologists that the liquid dosage forms of antibiotics (suspensions, syrups) are most effective [21]. Not conducive to eradication and subsequent, after a short period of time, eating. When we thought about the possibility of $\mathrm{Hp}$ eradication with a solution of drugs about 20 years ago, there was a lack of publications on this issue. Some researchers tried to apply aqueous solutions of crushed tablets (oral messages), but did not receive a significant increase in the efficiency of eradication of the infectious and therefore did not publish these results. On the basis of studies of the structure and functional properties of the wall layer of mucus of the digestive tract, it is known that the luminal surface of this layer is charged and hydrophobic and therefore opposes the diffusion of fluid into the depth of the layer. At the same time, when conducting a respiratory test for the diagnosis of $\mathrm{Hp}$ infection using stabilized isotope 13C-urea. dissolved in orange juice, the layer of gastric mucus does not oppose its rapid diffusion and interaction with Hp. The use of orange juice in this case was justified by the presence of citric acid in it, which delays the evacuation of fluid from the stomach, which lengthens the time of interaction of the infection with 13C-urea.

However, this effect is very weak, which suggests the presence of another reason that facilitates the diffusion of liquid and substances dissolved in the orange juice. Experimental testing on the opened stomachs of rats showed that the organic components of orange juice have an affinity for the surface of the mucin layer and, actively sorbing on it, neutralize the charge and make the surface of mucus hydrophilic. The only component of the juice that has such an affinity for the mucin gel of the mucus layer is gel solutions of pectins, the content of which in the juice is quite high (2.0-3.0g/l). With the unwillingness or intolerance of an orange juice patient, other unclarified juices (apricot, peach, plum) can be used, the content of pectins in which is comparable with orange, and the $\mathrm{pH}$ of citric and malic acids is about 4.5. To increase the time of interaction of the gastric mucosa with a solution of drugs, it is possible to use the topographic and anatomical features of the stomach and carry out an eradication procedure in a supine position on the left side when the pyloric channel is directed upwards, which makes it difficult to evacuate the liquid.

Like pectin, a colloidal bismuth substrate (De-nol) has a similar affinity for the surface of the mucus layer. About 25 years ago in the Central Research Institute of Gastroenterology L.I. Aruin, A.A. Ilchenko and V.B. Potapov studied the mechanism of interaction of bismuth colloidal sub-citrate (De-nol suspension) with the gastric mucosa. In the study in a scanning microscope, it was found that the supramolecular globules of the drug are sorbed on the surface of the sliz (personal communication, unpublished data), from which the colloidal bismuth subcitrate diffuses into the zone of $\mathrm{Hp}$ location and interacts with it. If the patient did not eat food after taking the drug, then the mentioned complexes remain on the surface of mucus even after 12 hours, which refutes allegations that the drug from the mucosal surface disappears almost completely after an hour [21]. It should not be forgotten that the preparation of bismuth colloidal sub-citrate (De-nol) when entering the market by Yamanouchi was produced in the form of a colloidal suspension, and not in tablets. Currently, in a number of countries, the release of bismuth colloidal sub-citrate in liquid form has been resumed, although in all reference books it is designated by the chemical formula as "Bismuth tri-potassium dicitrate".

Since Hp eradication is carried out most often in the treatment of peptic ulcer disease associated with this infection, accompanied, as a rule, by a painful symptom, preparations inhibiting the secretion of hydrochloric acid are included in the treatment regimen. Their choice should be justified by the properties of drugs that provide optimal treatment conditions, that is, rapid relief of pain, effective scarring of an ulcer defect, as well as the complete destruction of bacillary and coccal forms of $\mathrm{Hp}$ in the stomach. In relation to the relief of pain syndrome and $\mathrm{H}^{+} / \mathrm{K}^{+}$-ATPase inhibitors, and the $\mathrm{H} 2$ 
receptor blockers of the parietal cells to histamine are practically equivalent, about which there are numerous publications.

The rate of cicatrization of ulcers when performing adequate treatment depends on the size of the ulcers and, when eradication is achieved, $\mathrm{Hp}$ infections are the same for both groups of hydrochloric acid secretion inhibitors, whereas if the infection is preserved, the ulcers can heal faster for 4-7 days using $\mathrm{H} 2$ blockers receptor of parietal cells to histamine. This is clearly seen when the treatment begins at the peak of the development of an ulcer with bleeding [22]. Apparently, a more effective scarring with the use of histamine $\mathrm{H} 2$ receptor blockers depends on their antioxidant effect [23], a decrease in the production of reactive oxygen species by the infective itself [24], neutrophils own plate of the mucous membrane of the stomach, and, consequently, leads to a decrease in the activity of the inflammatory response. In addition, it should be noted that $\mathrm{H}^{+} / \mathrm{K}^{+}$-ATPase inhibitors, which inhibit hydrochloric acid production in the active phase of digestion (as well as the basal) by $85-95 \%$, inhibit the gastric phase of the proteolytic cascade and lead to a decrease in protein digestion by $25-30 \%$ [25]. This affects the deterioration of the provision of reparative processes plastic material. Considering the above, the use of histamine H2-receptor blockers in the treatment of peptic ulcer is more preferable.

To overcome the above reasons for the low efficacy of eradication therapy, we have proposed a new methodological approach based on the use of «liquid technology». It includes:

1. A four-part eradication regimen as a first-line therapy;

2. Inhibitors of histamine H2-receptors to reduce the secretion of hydrochloric acid and suppress the production of reactive oxygen species by infekt and neutrophils of the own plate of the gastric mucosa;

3. Antibacterial drugs and antibiotics, which are not produced resistance $\mathrm{Hp}$ and which can act in a wide range of $\mathrm{pH}$.

As means intended for eradication, we propose to include in the scheme only those that can actively influence the infection $(\mathrm{Hp})$, operate in a wide range of $\mathrm{pH}$, and for which resistance is not developed: amoxicillin (flemoxin solyutab) - 1000mg, nol - 240mg and enterofuril (nifuroxazide) - 400mg per dose. Of the nitrofuran preparations, preference should be given to enterofuril, which is produced in the form of a liquid emulsion. The above preparations, after fine grinding, are dissolved in 120-150ml of orange (or other unclarified) juice. A third-generation histamine $\mathrm{H} 2$ receptor blocker, famotidine (quamel) in a standard dosage of $20 \mathrm{mg}$, is included in the four-component antihelicobacter therapy scheme, which may not be used if eradication therapy is carried out during the interictal period of the disease.

The treatment procedure is as follows. An hour before meals (in the morning and in the evening) the patient takes a famotidine tablet (if necessary) with a small amount of water. After 30 minutes, drink the prepared solution of the above anti-helicobacter drugs in the juice. After taking the solution, the patient takes a lying position on the left side, turning every 2-4 minutes on the stomach, and again takes the previous position. It is also possible that the jerky pressure of the fingers of the right hand on the epigastric region of the anterior abdominal wall causes «splashing noise» in the stomach, facilitating the washing of the entire mucosal surface. After 30 minutes the patient can eat. Eradication therapy continues for one week. The control determination of $\mathrm{Hp}$ is carried out 4 to 6 weeks after the end of the administration of anti-helicobacter and antisecretory drugs. The effectiveness of treatment and reliable eradication of the infectious agent (HP) is achieved not only by exposing the entire surface of the gastric mucosa to the stomach mucosa for a long time (which is extremely important), but also due to overcoming the hydrophobicity of the mucus layer and ensuring active diffusion of the preparations deep into this layer directly into the persistence zone Infecta (Hp).

The obtained results showed a significant increase in the efficiency of eradication when using the liquid technology of antihelicobacter medications, the possibility of reducing the duration of therapy and a significant reduction in financial costs. Testing of liquid technology using the above four-component scheme in medical centers in Tver and Khabarovsk (more than 100 patients) confirms almost $100 \%$ eradication efficiency when using three methods of $\mathrm{Hp}$ infection verification before and after treatment. The results of testing in 2004 were reported at the European Helicobacter Congress in Vienna [26] and found the understanding and approval of BJ Marshall and D Grechem. The principle of fluid technology was repeatedly published in Russian domestic journals $[27,28]$, but was not accepted for publication in the journal Helicobacter.

Thus, the analysis of all five consensus testifies to the constant search for new schemes due to the existing dissatisfaction with the results of eradication therapy not only of the medical community, but also of the experts themselves. But, in fact, there are no radical changes. A certain deadlock has been formed, the way out of which should not be expected until 2020, when it is possible in the same Florence, experts will gather, preferably in the same composition and will discuss how clarithromycin is better than metronidazole. For now doctors will use the standards of the European recommendations.

\section{References}

1. (1994) NIH consensus development panel on Helicobacter pylori in peptic ulcer disease. NIH Consensus Development Panel on Helicobacter pylori in Peptic Ulcer Disease. JAMA 272(1): 65-69.

2. (1997) Current European concepts in the management of Helicobacter pylori infection. The Maastricht Consensus Report. European Helicobacter Pylori Study Group. Gut 41(1): 8-13.

3. (1993) Helicobacter pylori infection. Aspects of pathogenesis and therapy. In: Noach LA (Ed.), Tytgat GNJ, Amsterdam, Netherlands, p. 168.

4. Malfertheiner P, Megraud F, O’Morain C, Hungin AP, Jones R, et al. (2002) Current concepts in the management of Helicobacter pylori infectionThe Maastricht 2-2000 Consensus Report. Aliment Pharmacol Ther 16(2): 167-180.

5. Malfertheiner P, Megraud F, O’Morain C, Bazzoli F, El-Omar E, et al. (2007) Current concepts in the management of Helicobacter pylori infection: the Maastricht III Consensus Report. Gut 56(6): 772-781. 
6. Malfertheiner P, Megraud F, O'Morain CA, Atherton J, Axon AT, et al. (2012) Management of Helicobacter pylori infection-- the Maastricht IV / Florence Consensus Report. Gut 61(5): 646-664.

7. Graham DY, Fischbach L (2010) Helicobacter pylori treatment in the era of increasing antibiotic resistance. Gut 59(8): 1143-1153.

8. Malfertheiner P, Megraud F, O’Morain CA, Gisbert JP, Kuipers EJ, et al. (2017) Management of Helicobacter pylori infection-the Maastricht V/ Florence Consensus Report. Gut 66(1): 6-30.

9. Kaneko E, Hoshihara Y, Sakaki N, Harasawa S, Ashida K, et al. (2000) Peptic ulcer recurrence during maintenance therapy with $\mathrm{H} 2$-receptor antagonist following first-line therapy with proton pump inhibitor. J Gastroenterol 35(11): 824-831.

10. Schwartz H, Karause R, Sahba B, Herber M, Weissfeld A, et al. (1998) Triple versus dual therapy for eradicating Helicobacter pylori and preventing ulcer recurrence: a randomized, double-blind, multicenter study of lansoprazole, claritromycin, and/or amoxicillin in different dosing regimens. Am J Gastroenterol 93(4): 584-590.

11. Isakov VA, Domoradsky IV (2003) Helicobacter pyloriosis. Medpraktika M, Hyderabad, Telangana, India, p. 412.

12. Khomeriki SG, Khomeriki NM (2002) Hidden aspects of the clinical use of H2-blockers. Farmateka 9: 9-16.

13. Pasechnikov VD, Ivashkin VT, Chukov SZ (2002) Proton pump inhibitors in the treatment of precancerous diseases of the esophagus. In the book Prevention and treatment of chronic diseases of the upper gastrointestinal tract. M MEDpress-inform, Hyderabad, Telangana, India, pp. 96-124.

14. Pimanov SI (2000) Esophagitis, gastritis and peptic ulcer. Novgorod N, Ed, M Medical book, NGMA, p. 378

15. Khomeriki NM Khomeriki SG (2001) Experience in the use of fourcomponent drug regimens, other than standard quadrotherapy, in the treatment of Helicobacter pylori infection. Ros zh gatroenterol hepatol and Coloproctology 11(2): 103-105.

16. Khomeriki NM, Khomeriki SG (2004) Four-component schemes in the treatment of Helicobacter pylori infection: eradication without rehabilitation. Farmateka 13: 19-22.
17. Graham DY, Osato MS, Hoffman J, Opekun AR, Anderson SY, et al. (2000) Metronidazole containing Helicobacter pylori: a prospective study. Aliment Pharmacol Ther 14: 745-750.

18. Halperin Yu M, Lazarev PI (1986) Digestion and homeostasis. Nauka, Russia, p. 304

19. Krivova NA, Dambaev GTs, Khitrikheev VE (2002) The epepithelial mucous layer of the gastrointestinal tract and its functional significance. Tomsk p. 315 .

20. Endo H, Yoshida H, Ohmi N, Higuchi S (2001) Effects of lansoprazole and amoxicillin on uptake of [14C] clarithromycin into gastric tissue in rats. Antimicrob Agents Chemother 45(12): 3451-3455.

21. Leonova, MV, Belousov, Yu B (1996) H2-blockers in gastroenterological practice. M Gideon Richter p. 62.

22. Morozov IA, Lukina EV, Lopatina IV, Grinberg AA (1999) Dynamics of repair of duodenal ulcers after bleeding. In the book: "Helicobacter pylori: a revolution in gastroenterology. Tempus M p. 88-97.

23. Khomeriki S, Khomeriki N (2002) Hidden aspects of the clinical use of H2-blockers. Farmateka 9: 9-16.

24. Khomeriki SG, Zhukhovitsky VG, Khomeriki NM, Kubatiev AA (2000) Mechanisms of activation and suppression. Gut 47(Suppl.1): A7.

25. Morozov IA (2001) Structural aspects of the mechanism of action of H/KATPase blockers on the parietal cell in the treatment of Hp-associated diseases. Ross G Gastroenterol Hepatitis and Col 11(2): 68-70.

26. Morozov IA, Nikonov EL, Miller DA (2004) New liquid method for Helicobacter pylori eradication. Multicenter study. Helicobacter 9(5): 578-579.

27. Morozov IA, Nikonov EL, Miller DA (2012) Structural and functional substantiation of the optimal conditions for the eradication of Helicobacter pylori in the stomach. Evidence-based gastroenterology 1(2): 63-67.

28. Morozov IA (2015) Effective technology for the eradication of Helicobacter pylori infection in the stomach. Polyclinic Gastroenterology 2: 29-32.
Creative Commons Attribution 4.0 International License

For possible submissions Click Here

\section{Submit Article}

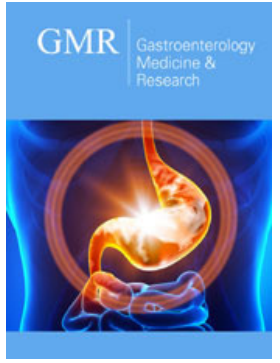

Gastroenterology Medicine \& Research

\section{Benefits of Publishing with us}

- High-level peer review and editorial services

- Freely accessible online immediately upon publication

- Authors retain the copyright to their work

- Licensing it under a Creative Commons license

- Visibility through different online platforms 